Nachruf

Z Rheumatol 2019 · 78:202

https://doi.org/10.1007/s00393-019-0608-9

(c) Springer Medizin Verlag GmbH, ein Teil von Springer Nature 2019
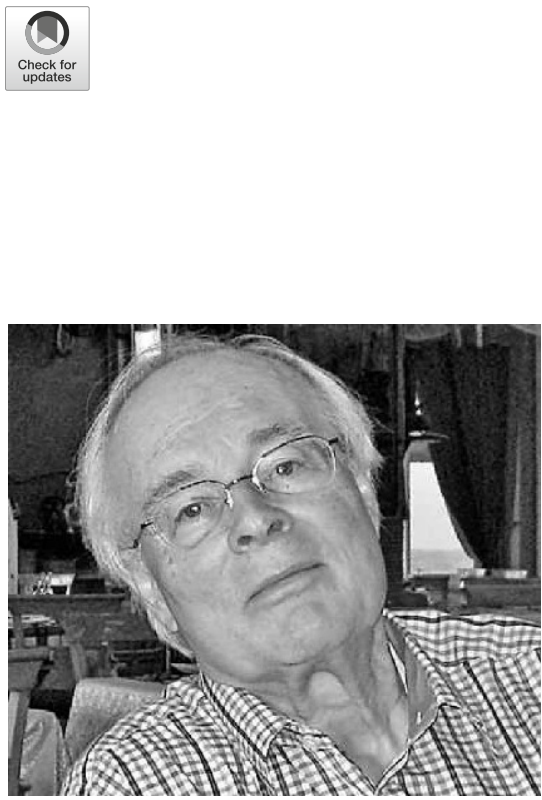

Am 08.12.2018 verstarb Professor Dr. med. Gerhard L. Bach aus Grünstadt (DÜW) im Alter von 84 Jahren an den Folgen einer schweren Erkrankung, deren operativer Behandlung er sich Anfang April 2017 hatte unterziehen müssen. Mit ihm, ein bis in sein hohes Alter von seinen Patienten geschätzter Arzt, ist ein großer klinischer Rheumatologe, international anerkannter Wissenschaftler und ein in wissenschaftlichen Fachgesellschaften engagierter Kollege von uns gegangen.

Gerhard L. Bach kam am 11. Juni 1934 in Dudenhofen (Pfalz) als Erstgeborener von Rudolf Bach und Ehefrau Gertrud (geb. Ofer) zur Welt. Nach dem Abitur am Humanistischen Gymnasium in Speyer studierte er ab 1954 Humanmedizin an der Ruprecht-Karls-Universität Heidelberg. Nach dem Staatsexamen, Erhalt der Approbation sowie der Promotion führte ihn sein weiterer beruflicher Weg 1962 nach Philadelphia, Pennsylvania, USA, um sich dort die Kenntnisse der damals noch jungen Disziplin Immunologie und Rheumatologie anzueignen, er erwarb den amerikanischen MD, wurde Dozent am Cook County Hospital, Chicago, Illinois, und später „Professor and Chairman“ des „Department of Clin-

M. Schattenkirchner ${ }^{1}$ K K. K. Förster ${ }^{2}$

'München, Deutschland

${ }^{2}$ Engelskirchen, Deutschland

\title{
Nachruf auf Prof. Dr. med. Gerhard L. Bach, MD (1934-2018)
}

ical Immunology and Rheumatology“ am Cook County Hospital, Chicago, Illinois.

In die Zeit des Amerikaaufenthaltes fällt die Eheschließung mit seiner Frau Marjorie („Gretl“), die ihm noch in den USA und später in Deutschland insgesamt fünfSöhne schenkte und nach vielen gemeinsamen Jahrzehnten bereits 2006 verstarb.

Nach seiner Rückkehr (1971) habilitierte sich Gerhard Bach an der LudwigMaximilian-Universität München mit einer Arbeit zur Serologie und biologischen Bedeutung des Rheumafaktors (1973), wurde wenig später Oberarzt (bei Prof. Mathies) im Rheumazentrum Bad Abbach und baute dort ein Immunlabor auf. 1977 wurde Prof. Bach Chefarzt und Leitender Medizinaldirektor der Rheumaklinik in Bayreuth und wenige Jahre später (1991) Chefarzt der „Klinik Auerbach“ in Bensheim, wo er neben der klinischen Betreuung vieler Patienten die wissenschaftliche Kooperation mit den Rheumazentren Heidelberg und „RheinMain“ (Frankfurt) pflegte. Seine akademische Lehrtätigkeit führte er weiterhin an der Universität Erlangen durch.

Sein über seine ärztliche Tätigkeit hinausgehendes Engagement für seine $\mathrm{Pa}$ tienten zeigt sich unter anderem in seiner Funktion als ehemaliger Präsident des Landesverbandes Bayern der Deutschen Rheuma-Liga, dessen Ehrenpräsident er nach seiner aktiven Zeit weiterhin war. Sein medizinisch-wissenschaftliches Engagement zeigt sich in der Betreuung einer Vielzahl von Assistenten und Doktoranden, in der Implementierung und jahrelangen Durchführung ärztlicher Fortbildungsveranstaltungen („Aktuelle Rheumatologie“). Er war wissenschaftlicher Herausgeber und/oder Bei- rat etlicher Fachzeitschriften und Autor, auch Mitautor, einer Vielzahl von Buchbeiträgen und Publikationen in Fachzeitschriften.

Die Verbundenheit von Prof. Bach - jahrelang Beirat der Deutschen Gesellschaft für Rheumatologie - mit ausländischen Kollegen zeigt sich in seiner Mitgliedschaft im „American College of Rheumatology“ (ACR), in seiner Mitgliedschaft in der "International Society for Rheumatic Therapy“ (ISRT), deren Kongresspräsident er 1992 war und „seinen“ Kongress damals in Mannheim durchführte, sowie in seiner Mitgliedschaft in der Ägyptischen Gesellschaft für Rheumatologie, die ihn zu Kongressvorträgen in Kairo und Alexandria, zusammen mit seiner zweiten Frau Erika Nathal-Bach, führten, zuletzt im Jahr 2011.

Unter den vielen Ehrungen sei pars pro toto das „Bundesverdienstkreuz 1. Klasse“ der Bundesrepublik Deutschland erwähnt, das Prof. Bach im Jahr 1995 für sein ärztliches Lebenswerk und sein Engagement für seine Patienten erhielt.

Wir trauern mit der Familie, tragen die Erinnerung an einen kompetenten und patientenzugewandten Arzt in uns und sind dankbar für eine jahrzehntelange Freundschaft.

Prof. Dr. med. Manfred Schattenkirchner (München)

Dr. Dr. h.c. Klaus K. Förster

(Engelskirchen)

\section{Korrespondenzadresse}

Dr. Dr. h.c. K. K. Förster

Igelweg 3, 51766 Engelskirchen, Deutschland kkfoerster@aol.com 